\title{
Reflets
}

Revue ontaroise d'intervention sociale et communautaire

\section{Défis dans l'évaluation du bégaiement chez les bilingues}

\section{Patricia M. Roberts}

Volume 8, numéro 1, printemps 2002

La réadaptation : son visage français en Ontario

URI : https://id.erudit.org/iderudit/026372ar

DOI : https://doi.org/10.7202/026372ar

Aller au sommaire du numéro

Éditeur(s)

Reflets : Revue ontaroise d'intervention sociale et communautaire

ISSN

1203-4576 (imprimé)

1712-8498 (numérique)

Découvrir la revue

Citer cet article

Roberts, P. M. (2002). Défis dans l'évaluation du bégaiement chez les bilingues. Reflets, 8(1), 47-60. https://doi.org/10.7202/026372ar

Tous droits réservés (C) Reflets : Revue ontaroise d'intervention sociale et communautaire, 2002
Ce document est protégé par la loi sur le droit d'auteur. L'utilisation des services d’Érudit (y compris la reproduction) est assujettie à sa politique d'utilisation que vous pouvez consulter en ligne.

https://apropos.erudit.org/fr/usagers/politique-dutilisation/ 


\section{Défis dans l'évaluation du bégaiement chez les bilingues}

\section{Patricia M. Roberts}

École des sciences de la réadaptation (audiologie et orthophonie)

Université d'Ottawa

\section{L'évaluation en orthophonie}

L'évaluation du bégaiement porte sur plusieurs plans dont l'histoire du cas (évolution du trouble, développement linguistique et moteur), l'impact du trouble sur la qualité de vie et la thérapie antérieure. On cherche aussi à mesurer la sévérité du bégaiement, à dresser un inventaire des symptômes, en comparant la parole du client bègue à celle des non-bègues.

Pour déterminer si la parole d'un individu qui bégaie est dans les limites de la normale, l'orthophoniste enregistre le client en train de parler et de lire à haute voix. On analyse ensuite cet échantillon pour le comparer aux données normatives. On analyse plusieurs aspects de la parole et des disfluidités, c'est-à-dire répétitions de parties de mots, blocages, prolongations (leur durée, la tension associée à leur articulation, par ex.). Deux dimensions de la parole retiennent particulièrement l'attention des chercheurs : le débit, calculé en mots par minute ou en syllabes par minute, et le taux de disfluidité, souvent calculé comme nombre de disfluidités dans 100 syllabes. Ce sont aussi les deux aspects de la parole étudiés dans la recherche que nous présenterons ci-dessous.

Tous les locuteurs produisent des disfluidités en parlant. Bon nombre d'études ont montré que, dans la parole des anglophones 
non-bègues, se retrouvent des interjections (par ex. en anglais uh..., well... you know et en français euh... tiens... là ), des faux départs (commencer un mot ou une phrase que l'on modifie ensuite, par ex. "je cr- je pense que.....»; « il fait - il a fait ....»). Il y a aussi des répétitions de mots ou de plusieurs mots. Dans la parole des personnes bègues, on trouve ces mêmes disfluidités, mais aussi d'autres types qui sont rares chez les non-bègues. Des répétitions de parties de mots, des moments de tension où l'individu semble bloquer et des sons prolongés (ex.jjijjijjije dois pa-pa-partir) sont typiques de la parole bègue, mais rares dans celle des locuteurs non-bègues. Ces disfluidités sont si rares dans la parole des anglophones non-bègues et si fréquentes dans celles des anglophones bègues qu'on les a nommées "disfluidités de type bégaiement » (Stuttering-Like Disfluencies) (Yairi et Ambrose 1992).

Dans l'évaluation des clients anglophones, on se base sur des études décrivant la parole normale. Elles ne sont pas nombreuses. Cependant, nous y trouvons des données qui servent de points de repère (Johnson 1961;Andrews et Ingham 1971; Lutz et Mallard 1986; Duchin et Mysak 1987; Walker 1988;Venkatagiri 1999). Il y a beaucoup moins de recherches pertinentes portant sur les francophones et, à ma connaissance, aucune ne touche les disfluidités dans la parole des adultes bilingues.

Les disfluidités sont présentes dans la parole en espagnol (Johnson, O'Connell, et Sabin 1979), en portugais (Naro et Scherre 1996), en japonais (Kawakami et Ito 1999), en Igbo (langue parlée en Nigéria) (Nwokah 1988) et en français (Grosjean et Deschamps 1975). Il s'agit probablement d'un phénomène universel. Par contre, nous ne savons pas dans quelle mesure le nombre et la fréquence des différents types de disfluidités varient d'une langue à l'autre. Des recherches récentes réalisées à l'Université d'Ottawa et résumées ci-dessous, démontrent l'importance d'étudier différentes langues. Nos résultats indiquent que les francophones, qu'il soient unilingues ou bilingues, ont un débit et un taux de disfluidité différents de ceux des anglophones et que les types de disfluidités ne sont pas toujours les mêmes. 
"Les outils

d'évaluation

disponibles en français

sont peu nombreux, et

très peu parmi ceux

qui existent ont des

normes appropriées

pour le Canada.»
Les outils d'évaluation disponibles en français sont peu nombreux, et très peu parmi ceux qui existent ont des normes appropriées pour le Canada (Garcia et Desrochers 1997). Cette lacune se fait surtout sentir par rapport aux troubles du langage (le vocabulaire, la syntaxe ou la lecture) et aux troubles de l'articulation (pour enfants avec retards ou difficultés dans l'apprentissage des sons de la langue maternelle). Dans l'évaluation du bégaiement, l'orthophoniste utilise assez rarement des outils d'évaluation standardisés, surtout face à des clients adultes. L'évaluation se fait, par contre, à la lumière des données disponibles sur le débit et les disfluidités. Au Canada, ces données existent pour l'anglais, mais pas pour le français, ce qui apporte plusieurs défis à l'orthophoniste qui doit évaluer les adultes bègues bilingues. Notre recherche récente tente d'explorer des questions sur le débit et les disfluidités. Les premiers résultats que nous obtenons démontrent l'importance de ce thème de recherche pour l'orthophonie, tant sur le plan de l'évaluation que sur celui de l'intervention.

\section{Quel est le débit de parole des francophones non- bègues?}

On ne le sait pas. Pour les locuteurs anglophones, plusieurs études établissent des moyennes et des étendues du débit normal en parole spontanée et en lecture à voix haute. Un clinicien utilise de deux façons au moins les données sur le débit des adultes non-bègues. En y comparant la parole de son client, l'orthophoniste peut dire à quel point le bégaiement ralentit le débit. Plus le débit est lent par rapport à la norme, plus la parole sera perçue comme anormale. Les personnes bègues qui débitent trop lentement un message au téléphone s'exposent régulièrement au ridicule et aux remarques désobligeantes. Aussi, dans la thérapie, on peut cibler un débit "normal " en se basant sur ces données.

Pour les anglophones, plusieurs études concluent à un débit se situant autour de 200 syllabes par minute en conversation ou 
"Constituer des normes pour les francophones au Canada ne sera pas tâche facile. D'une province à une autre, il $y$ a plusieurs variantes régionales du français d'un individu à l'autre, différents niveaux de bilinguisme.» en monologue. Par exemple,Andrews et Ingham (1971) proposent une étendue variant de 162 à 230 syllabes par minute. Culatta et Leeper (1989) ont testé des adultes américains âgés de 25 à 34 ans. Le débit moyen était de 188 syllabes par minute avec un écart-type de 44. Walker (1988) trouve une moyenne d'environ 230 syllabes par minute.

Pour les francophones, il n'existe aucune recherche clinique portant sur cette question. Les quelques études du débit ont utilisé des interviews avec des politiciens ou des comédiens à la radio (Grosjean et Deschamps 1975) et ont testé des francophones européens. Pour le Canada français, il ne semble pas y avoir d'études. Donc, le clinicien doit ou bien porter un jugement subjectif quant au débit, ou bien utiliser les données portant sur l'anglais.

Constituer des normes pour les francophones au Canada ne sera pas tâche facile. D'une province à une autre, il y a plusieurs variantes régionales du français d'un individu à l'autre, différents niveaux de bilinguisme. Ce dernier point revêt une grande importance parce qu'il semblerait que le débit est influencé par le niveau de bilinguisme et par le fait que le bilinguisme est la norme dans beaucoup de régions.

Pour évaluer la sévérité d'un trouble, on doit comparer la personne bègue à ses pairs. La recherche normative en psycholinguistique sépare typiquement les participants en groupes selon leur âge et leur niveau de scolarité. Pour arriver à des normes pour la population bilingue, il faudra avoir des sous-groupes selon le type et le niveau de bilinguisme, donc un plus grand nombre de sujets pour pouvoir constituer plus de sous-groupes.

En Ontario, la majorité des francophones sont, jusqu'à un certain point, bilingues. Cela ne veut pas dire qu'ils maitrisent parfaitement les deux langues. Rares sont les individus qui atteignent cet idéal. Le bilinguisme est un continuum. Chaque individu se situe à différents points sur ce continuum. Il ne s'agit pas d'un seul niveau pour chaque individu, parce que le niveau de bilinguisme varie selon la modalité linguistique : compréhension orale, expression orale, lecture et expression écrite (Roberts 2001). Aussi, les deux langues s'influencent l'une l'autre d'une façon 
plus ou moins grande selon certains facteurs, dont le niveau de maîtrise de la langue, la ressemblance entre les deux langues parlées et l'âge d'acquisition de chacune d'elles. Selinker (1992) offre une bonne synthèse de la littérature sur le sujet.

Pour explorer l'impact du bilinguisme sur le débit, Roberts et Hébert (2001) ont testé à Moncton 19 enfants francophones âgés de 9 ou 10 ans. Dix se sont dits équilingues, avec un niveau de maîtrise égal du français (langue maternelle) et de l'anglais alors que 9 ont dit mieux maitriser le français que l'anglais. Il n'y a pas eu de différence significative dans le débit des deux langues pour le groupe équilingue (166,7 syllabes par minute en français et 158,9 en anglais). Mais pour le groupe non-équilingue, le débit était plus rapide en français, la langue forte, qu'en anglais : 180,2 contre 131,0 syllabes par minute respectivement (test $t, p<, 001$ ).

Une étude de 4 adultes bègues démontre aussi que le débit semble varier selon le niveau de maitrise de chaque langue (Roberts 2002). Deux hommes équilingues (français/anglais) ont parlé à un débit semblable dans les deux langues, alors que deux femmes ont parlé plus vite en français (leur langue préférée) qu'en anglais.

\section{Quel est le taux de disfluidité normale dans la parole des francophones canadiens non-bègues?}

Encore une fois, les données connues portent surtout sur l'anglais. Une étude américaine rapporte que les jeunes adultes produisent en moyenne 6,5 disfluidités pour 100 mots (Johnson 1961), et une autre a trouvé un résultat semblable, soit 7,4 (Duchin et Mysak 1987). Il est probable que ce niveau de disfluidité serait normal pour les hommes canadiens anglophones. Roberts, Meltzer et Wilding (2001) ont testé 25 Canadiens anglophones. Dans des monologues sur leur emploi (le sujet le plus utilisé dans ce genre d'évaluation clinique), la moyenne était de 6.9 disfluidités dans 100 syllabes, avec une étendue de 2.3 à 13.0. 
«...utiliser les

résultats des

anglophones pour

évaluer jusqu'à quel

point le taux de disfluidité d'un client francophone est anormal mènerait à une sur-estimation de la sévérité du trouble.»
Et les francophones? Des étudiantes en orthophonie de l'Université d'Ottawa viennent de compléter une étude qui reprend celle de Roberts, Meltzer et Wilding (2001), mais auprès d'adultes francophones. Une trentaine d'hommes de Montréal, de l'Outaouais et d'Ottawa y ont participé. Les résultats préliminaires sont étonnants. Le taux de disfluidité est presque le double de celui des anglophones : moyenne de 13.8 disfluidités dans 100 syllabes, avec une étendue de 5 à 23 . Le taux de disfluidité en français semble être le même pour les locuteurs unilingues et bilingues.

Selon ces résultats préliminaires, les francophones sont beaucoup plus disfluides que les anglophones. Donc, utiliser les résultats des anglophones pour évaluer jusqu'à quel point le taux de disfluidité d'un client francophone est anormal mènerait à une sur-estimation de la sévérité du trouble.

Ce résultat ne s'applique pas à tous les francophones. On sait que beaucoup de paramètres de la parole varient selon le niveau de bilinguisme. Dans quelle langue produit-on plus de disfluidités - dans sa langue maternelle (L1) ou dans sa deuxième langue (L2)? Dans sa langue forte ou dans une langue faible? Ces questions importantes n'ont pas encore été abordées. L'étude de Roberts et Hébert (2001) souligne l'importance de cette question quand les 10 enfants équilingues testés ont parlé avec un taux de disfluidité presque égal dans les deux langues : 7,5 disfluidités dans 100 syllabes en français et 6,7 en anglais. Par contre, les 9 enfants qui ont dit mieux maîtriser le français ont mieux parlé dans cette langue : 8,4 disfluidités dans 100 syllabes en français, mais 10,8 en anglais $(\mathrm{p}<, 054)$. En interprétant ce résultat, non-significatif, il faut tenir compte du fait que le nombre de sujets est petit et que le niveau normal de disfluidité serait plus élevé en français qu'en anglais. Donc, la différence observée entre les 2 langues a peutêtre plus d'importance que la valeur de $\mathrm{p}$ ne semble indiquer. 


\section{Quels types de disfluidités sont typiques du français des non-bègues?}

À nouveau, nous ne le savons pas. À écouter parler francophones et anglophones, on constate que les mêmes types de disfluidités sont présents : les interjections, les faux départs et les répétitions de mots font partie de la parole de tous.

Mais, des recherches permettent de croire que la fréquence des différents types varie d'une langue à l'autre. Nwokah (1988) dit qu'en Igbo on fait parfois une prolongation là où l'anglais mettrait une interjection. Grosjean et Deschamps (1975) notent le même phénomène pour le français en Europe. L'étude en cours des adultes francophones fournira des données précises portant sur cette question. En parcourant les données, on constate que le taux de prolongation des sons est beaucoup plus élevé dans les monologues des francophones que dans ceux des anglophones testés par Roberts, Meltzer et Wilding (2001).

Pourquoi serait-il important de mesurer la présence de prolongations dans la parole des francophones non-bègues? En anglais, elles sont très rares et sont vues comme étant un symptôme du bégaiement. Sans données pour les francophones, on risque d'attribuer au bégaiement des prolongations qui sont tout à fait normales.

\section{L'effet d'adaptation}

Même lorsque nous aurons les données nécessaires sur le débit et le taux de disfluidité, il restera un autre défi à surmonter. Dans l'évaluation clinique, on doit évaluer la parole dans toutes les langues qu'un client utilise dans sa vie courante. Il y a lieu de se demander dans quelle mesure la répétition des mêmes tâches dans différentes langues entrave la validité des résultats obtenus. 
Il est reconnu que la lecture répétée d'un texte amène un débit plus rapide et une baisse dans le nombre de moments de bégaiement. Ce phénomène s'appelle l'effet d'adaptation (Silverman 1996). Il est observé non seulement en lecture, mais aussi lorsqu'un locuteur décrit de façon répétée un événement ou un objet. Or, dans l'évaluation de la parole des personnes bègues, on demande au client de décrire son travail (ou ses études). On pourrait choisir le même thème pour les deux langues et obtenir les résultats suivants :

- Français :

22 disfluidités dans 100 syllabes, débit de 182 syll/min., $1^{\text {ère }}$ langue testée;

- Anglais :

12 disfluidités dans 100 syllabes, débit de 180 syll/min., $2^{\mathrm{e}}$ langue testée.

Comment doit-on interpréter ces chiffres? Est-ce que le client parle de façon plus fluide en anglais, ou est-ce que l'anglais bénéficie de l'effet d'adaptation? Pour fins de cet exemple, on fait abstraction du problème présenté plus haut touchant le niveau normal de disfluidité qui ne semble pas le même pour les deux langues.

Une solution serait de varier le thème du discours d'une langue à l'autre. Mais, est-ce que le taux de disfluidité est uniforme pour différents thèmes? S'il ne l'est pas, on substitue, au problème d'adaptation, un problème de thème en changeant de thème lorsqu'on change de langue. On sait que la sévérité du bégaiement varie selon la personne à qui l'on s'adresse, le nombre d'interlocuteurs, les émotions ressenties par le locuteur (Silverman 1996). Est-ce que la sévérité varie aussi selon le thème d'un monologue? Nous avons comparé les taux de disfluidité des adultes non-bègues dans 3 monologues (Roberts, Meltzer et Wilding 2001). Les 3 thèmes étaient le travail ou les études, les activités de loisir et comment pratiquer un sport. Le nombre de disfluidités dans 100 syllabes n'a pas varié de façon significative selon le thème $(\mathrm{p}>, 05)$ pour un échantillon de 500 syllabes. Les taux de disfluidité étaient de 6,52, 7,56 et 6,98 par 100 syllabes. Reste à voir si cette 
stabilité se trouve également dans les monologues des francophones non-bègues et ensuite dans ceux des bègues.

À l'effet d'adaptation possible, il faut ajouter celui de la variabilité test-retest. Nous ne savons pas jusqu'à quel point le taux de disfluidité varie d'un échantillon à l'autre, ni d'un jour à l'autre. Est-ce qu'on peut minimiser cette variabilité en testant les deux langues des clients dans une seule séance? Est-ce qu'au contraire cette démarche aurait comme effet de masquer des différences entre les langues en maximisant l'effet d'adaptation (Roberts 2002)?

\section{Niveau de bilinguisme}

Même si on trouve que le taux de disfluidité ne varie pas selon le thème et, même lorsque nous saurons comment compenser pour la variabilité test-retest, nous ne pourrons pas nécessairement utiliser ces thèmes dans les deux langues des clients bègues. Le choix des thèmes doit respecter les domaines d'utilisation de chaque langue. Un bilingue a rarement tout son vocabulaire en double (Grosjean 1989; Kohnert, Hernandez, et Bates 1998; Roberts 2001). On peut, par exemple, utliser davantage l'anglais au travail et le français à la maison. Donc, on ne connait pas nécessairement le vocabulaire français relié au travail, ni les noms anglais de tous les meubles, aliments et jouets de ses enfants. Avant de choisir des thèmes pour les échantillons de parole, l'orthophoniste doit savoir quelle langue est utilisée dans quels domaines par le client.

On voudrait connaître le niveau de maitrise de chaque langue parce qu'il semblerait que l'on parle plus lentement et/ou que l'on produise plus de disfluidités dans sa langue faible (Raupach 1980; Roberts et Hébert 2001). En recherche, on demande souvent aux participants d'une étude de groupe de procéder à une autoévaluation de leur bilinguisme, en cotant de 1 à 7 leur compréhension orale et écrite, et leur expression verbale et écrite. 
Suite à cette auto-évaluation, on peut répartir les participants en groupes (niveau élevé ou niveau bas de bilinguisme). Par contre, nous avons peu de données appuyant la validité de cette démarche pour l'évaluation d'individus et aucune étude de la fluidité des locuteurs bilingues, non-bègues ne l'a utilisée.

Le fait que le bilingue n'utilise pas ses deux langues dans toutes les situations de la vie courante peut également compliquer l'évaluation de la lecture. Lorsqu'on évalue le bégaiement, on compare le niveau de disfluidité et le débit en parole (en monologue et/ou en conversation) au niveau de disfluidité en lecture. Cette comparaison permet de déceler un phénomène très fréquent, à savoir l'évitement de sons ou de mots. Dans la parole libre, la personne bègue peut choisir ses mots de façon à éviter des mots sur lesquels elle s'attend à bégayer. Elle peut aussi utiliser les interjections ou des à-côtés pour retarder l'usage de ces mots, se donnant le temps de mieux les préparer. L'utilisation de ces stratégies a pour effet de diminuer le nombre de disfluidités de type bégayé. Dans la lecture, ces stratégies ne sont plus possibles. On doit lire le texte sans rien y changer. Si les taux de disfluidité sont pareils en conversation et en lecture, l'orthophoniste conclut que le client n'utilise pas souvent des "trucs » d'évitement. Par contre, lorsque la différence entre ces deux tâches est marquée, il conclut que le taux de disfluidité en parole ne révèle pas toute la sévérité du trouble. Ainsi, l'élimination de ces stratégies devra faire partie de la thérapie.

\section{Démarches en thérapie pour les bilingues}

Une fois l'évaluation complétée, si le client est prêt à travailler pour de longs mois, on entame une thérapie. Par un processus de façonnement de la parole, de modification du comportement et de counselling, on refait la parole de l'adulte bègue. On le munit d'outils pour atteindre un meilleur niveau de fluidité dans la parole et pour se sortir des moments de disfluidité de façon à minimiser l'impact de ceux-ci sur la communication. Les méthodes les plus 
utilisées sont appuyées par des études qui démontrent leur efficacité mais, rappelons-le, surtout auprès d'anglophones. Plusieurs des méthodes actuelles sont décrites dans Curlee (1998).

Pour ce qui est de l'intervention chez les bilingues, il y a plusieurs études qui portent sur les enfants (Druce, Debney et Byrt 1997; Waheed-Khan 1998; Humphrey, Al Natour et Amayreh 2001). On trouve que la thérapie donnée dans une langue se généralise à l'autre pour la majorité des jeunes bilingues. L'amélioration dans la langue non-visée n'est pas nécessairement aussi grande que celle dans la langue de thérapie. Mais chez l'enfant, il y a une plus grande plasticité neurologique que chez l'adulte. L'acquisition des langues n'est pas complétée (Harley et Wang 1997; Birdsong et Molis 2001). Chez les adultes, on ne sait pas si la même généralisation d'une langue à l'autre se produira. Mais il semble que la généralisation des acquis peut se faire chez l'adulte. Une étude de cas d'une femme bilingue (espagnol/anglais) démontre un transfert des acquis. Cependant, la langue visée en thérapie s'est améliorée plus que la langue non-visée (Humphrey 1999).

Nous avons un besoin pressant de recherches portant sur l'intervention auprès d'adultes bègues pour voir dans quelle mesure cette généralisation a lieu et quels facteurs l'influencent (par ex., l'âge, les ressemblances entre les paires de langues, les méthodes de thérapie utilisées...). Dans la mesure où les acquis ne se généralisent pas d'une langue à l'autre, le client aura besoin de thérapie dans les deux langues. Pour ce faire, l'orthophoniste devra très bien maîtriser les deux langues. Selon la politique de l'American Speech-Language-Hearing Association, on peut offrir des services dans une langue seulement si on la possède à un niveau "langue maternelle ». Les associations canadiennes n'ont

«...on doit recruter

des cliniciens

équilingues pour répondre aux besoins des clients bilingues." malheureusement pas toutes une politique sur cette question. Donc, on doit recruter des cliniciens équilingues pour répondre aux besoins des clients bilingues. Aussi, dans la mesure où les acquis ne se généralisent pas d'une langue à l'autre, la thérapie prendra plus de temps et coûtera plus cher au système de santé. 


\section{Conclusion}

Les défis sont donc nombreux pour les orthophonistes qui doivent évaluer le bégaiement des bilingues. Si les projets décrits ci-haut servent déjà en milieu clinique pour guider les démarches auprès de clients, ils ne sont qu'à leurs débuts.

Nous avons besoin de recherches portant sur le français et sur les francophones bilingues au Canada. À partir des exemples fournis ci-haut, on voit aussi à quel point cette recherche sera compliquée. On devra tenir compte des variantes régionales, de la grande variabilité d'une personne bilingue à une autre, de multiples aspects du bilinguisme et du bégaiement ainsi que des interactions possibles entre chacun de ces facteurs.

\section{Références}

ANDREWS, Gavin et INGHAM, Roger J. (1971). «Stuttering : Considerations in the evaluation of treatment ", British Journal of Disorders of Communication, vol. 6, 129-138.

ANDREWS, Gavin., CRAIG, A., FEYER, A.M. et coll. (1983). «Stuttering : A review of research findings and theories circa 1982 ", Journal of Speech and Hearing Disorders, 48:3, 226-246.

BIRDSONG, David et MOLIS, Michelle (2001). «On the evidence for maturational constraints in second-language acquisition ", Journal of Memory \& Language, 44:2, 235-249.

CULATTA, Richard et LEEPER, L. (1989). Présenté dans Culatta, Richard et Goldberg, Stanley. (1995). Stuttering therapy: An integrated approach to theory and practice. Needham Heights, MA,Allyn \& Bacon, 76.

CURLEE, Richard F. éd. (1998). Stuttering and related disorders of fluency, New York, Thieme Medical Publishers.

DE NIL, Luc F. (1999). "Stuttering : A neurophysiological perspective », Nan Bernstein Ratner et Charles Healey, éds., Stuttering research and practice: bridging the gap, Mahwah, NJ, Lawrence Erlbaum Associates, 85-102.

De JOHNSON, Theresa, O'CONNELL, Daniel C. et SABIN, Edward J. (1979). «Temporal analysis of English and Spanish narratives » Bulletin of the Psychonomic Society, 13:6, 347-350.

DRUCE, Tanya, DEBNEY, Sue et BYRT,Ted (1997). " Evaluation of an intensive treatment program for stutteing in young children ", Journal of Fluency Disorders, 22:3, 169-186.

DUCHIN, Sandra W., et MYSAK, Edward D. (1987). « Disfluency and rate characteristics of young adult, middle aged, and older males ", Journal of Communication Disorders, 20:3, 245-257. 
FOX, Peter T., INGHAM, Roger J., INGHAM, Janice C., ZAMARRIPA, Frank, XIONG, Jin-Hu et LANCASTER, Jack L. (2000). «Brain correlates of stuttering and syllable production - A PET performance-correlation analysis ", Brain, 123(Part 10), 1985-2004.

GARCIA, Linda J. et DESROCHERS, Alain. (1997). «L'évaluation des troubles du langage et de la parole chez l'adulte francophone ", Revue d'orthophonie et d'audiologie, 21:4, 271-293.

GROSJEAN, François (1989). " Neurolinguists beware! The bilingual is not two monolinguals in one person ", Brain and Language, 36:1, 3-15.

GROSJEAN, François et DESCHAMPS, Alain (1975). «Analyse contrastive des variables temporelles de l'anglais et du français: vitesse de parole et variables composantes, phénomènes d'hésitation ", Phonetica, 31, 144-184.

HARLEY, Birgit et WANG, Wenxia (1997). «The critical period hypothesis :Where are we now? ", Annette B. de Groot et Judith F. Kroll, éds., Tutorials in bilingualism. Mahwah, NJ, Lawrence Erlbaum Associates, 19-52.

HUMPHREY, Brian D. (1999, novembre). Bilingual stuttering : Can treating one language improve fluency in both?, Communication affichée présentée au congrès annuel de l'American Speech Language and Hearing Association. San Francisco.

HUMPHREY,Brian D.,AL NATOUR,Yaser,AMAYREH, Mousa (2001, mai). Bilingual stuttering : comparing treatment studies of children vs. adults. Présenté au congrès de la Florida Speech and Hearing Association. Orlando, Floride.

JOHNSON, Wendell (1961). "Measurements of oral reading and speaking rate and disfluency of adult male and female stutterers and non stutterers ", Journal of Speech and Hearing Research, Monograph Supplement, 7, 1-20.

KAWAKAMI, Masayo, et ITO, Tomohiko (1999). Japanese Journal of Special Education, 37:2, 23-30.

KOHNERT, Kathryn J., HERNANDEZ, Arturo, E. et BATES, Elizabeth (1998). "Bilingual performance on the Boston Naming Test : Preliminary norms in Spanish and English ", Brain and Language, 65:3, 422-440.

LUTZ, Konnie C. et MALLARD, A.R. (1986). "Disfluencies and rate of speech in young adult non-stutterers ", Journal of Fluency Disorders, 11:4, 307-316.

NARO, Anthony J. et SCHERRE, Maria (1996). "Disfluencies in the analysis of speech data ", Language Variation and Change, 8:1,1-12.

NWOKAH, Evangeline E. (1988). "The imbalance of stuttering behavior in bilingual speakers ", Journal of Fluency Disorders, 13:5, 357-373.

RAUPACH, Manfred (1980). «Temporal variables in first and second language speech production », Hans W. Dechert et Manfred Raupach, éds. Temporal variables in speech, The Hague : Mouton Publishers, 263-270.

ROBERTS, Patricia M. (2001). "Aphasia assessment and treatment for bilingual and culturally diverse patients ", Roberta Chapey, éd. Language intervention strategies in aphasia and related neurogenic communication disorders. $4^{e}$ édition. Baltimore: Lippincott, Williams \& Wilkins, 208-232.

ROBERTS, Patricia M. (2002). «Disfluency patterns in four bilingual adults who stutter », Revue de l'audiologie et d'orthophonie, 26:1, 5-19.

ROBERTS, Patricia M. et HÉBERT, Tanya (2001, août). Le débit et les disfluidités chez des enfants francophones bilingues (Rate of speech and disfluencies in bilingual Francophone children). 
Présenté au $25^{\mathrm{e}}$ congrès mondial de l'Association internationale de logopédie et phoniatrie. Montréal.

ROBERTS, Patricia M., MELTZER, Ann et WILDING, Joanne (2001, août). Normal speech disfluencies across sample lengths and topics. Présenté au $25^{\mathrm{e}}$ congrès mondial de l'Association internationale de logopédie et de phoniatrie. Montréal.

SELINKER, Larry (1992). Rediscovering interlanguage. New York, Longman.

SILVERMAN, Franklin H. (1996). Stuttering and other fluency disorders : An overview for beginning clinicians. $2^{\mathrm{e}}$ éd. Needham Heights, MA, Allyn \& Bacon.

VENKATAGIRI, H.S. (1999). "Clinical measurement of rate of reading and discourse in young adults ", Journal of Fluency Disorders, 24:3, 209-226.

WAHEED-KHAN, N. (1998). "Fluency therapy with multilingual clients », E. Charles Healey et Hermann F.M. Peters, éds., Second world congress on fluency disorders : Proceedings, Nijmegen, Nijmegen University Press, 200-204.

WALKER, Virginia G. (1988). « Durational characteristics of young adults during speaking and reading tasks ", Folia phoniatrica, 40:1, 12-20.

WEBSTER, Ronald L. (1978). Precision Fluency Shaping Program : Clinician's Program Guide. Roanoke, VA : Communications Development Corporation.

WEBSTER, Ronald L. (1979). " Empirical considerations regarding stuttering therapy ", Hugo Gregory ,éd. Controversies about stuttering therapy. Baltimore : University Park Press, 209-239.

YAIRI, Ehud et AMBROSE, Nicoline (1992). "A longitudinal study of stuttering in children : A preliminary report ", Journal of Speech and Hearing Research, 35:4, 755-760. 